Daniel Weidner · Sigrid Weigel (Hrsg.)

Benjamin-Studien 2 

Daniel Weidner · Sigrid Weigel (Hrsg.)

Benjamin-Studien 2

Wilhelm Fink 
Bibliografische Information der Deutschen Nationalbibliothek

Die Deutsche Nationalbibliothek verzeichnet diese Publikation in der Deutschen Nationalbibliografie; detaillierte bibliografische Daten sind im Internet über http://dnb.d-nb.de abrufbar.

Alle Rechte, auch die des auszugsweisen Nachdrucks, der fotomechanischen Wiedergabe und der Übersetzung, vorbehalten. Dies betrifft auch die Vervielfältigung und Übertragung einzelner Textabschnitte, Zeichnungen oder Bilder durch alle Verfahren wie Speicherung und Übertragung auf Papier, Transparente, Filme, Bänder, Platten und andere Medien, soweit es nicht $\$ \$ 53$ und 54 UrhG ausdrücklich gestatten.

(C) 2011 Wilhelm Fink Verlag, München

(Wilhelm Fink GmbH \& Co. Verlags-KG, Jühenplatz 1, D-33098 Paderborn)

Internet: www.fink.de

Die Drucklegung dieses Werkes wurde unterstützt mit den Mitteln des Bundesministeriums für Bildung und Forschung unter dem Förderkennzeichen 1UG0712.

Lektorat: Bettina Moll, Berlin

Satz: Tilo Lothar Rölleke, Berlin

Einbandgestaltung: Evelyn Ziegler, München

Printed in Germany

Herstellung: Ferdinand Schöningh GmbH \& Co. KG, Paderborn

ISBN 978-3-7705-5071-5 


\title{
Die Bestimmung der Aufnahme Licht und Graphie bei Walter Benjamin ${ }^{1}$
}

\author{
Die Analphabeten der Zukunft und \\ das ABC kommender Lichtgestaltung
}

»Nicht der Schrift-, sondern der Photographieunkundige wird, so hat man gesagt, der Analphabet der Zukunft sein« (GS II, 385) - eingängig wie ein Slogan steht diese Formulierung im letzten Absatz von Walter Benjamins Kleiner Geschichte der Photographie. Der Autor dieser von Benjamin derart wiedergegebenen und gleichzeitig durch den Einschub sso hat man gesagt rhetorisch abgebremsten Parole ist ursprünglich László Moholy-Nagy, der 1927 in der Avantgardezeitschrift $i 10$ prognostiziert hatte, dass »die fotografie [...] in der nächsten periode ein unterrichtsfach wie heute das abc und das einmaleins« sein werde, denn es sei "der fotografie-unkundige der analfabet der zukunft «. ${ }^{2}$ Moholy präsentiert hier ein Narrativ, das Marshall McLuhan später in ähnlicher Form als Geschichte vom Ende der Gutenberg-Galaxis erzählen wird und das auch schon Moholys und Benjamins Zeitgenosse Béla Balázs im Rahmen seiner Stummfilmtheorie in ähnlicher Fassung angeboten hatten, indem er die Verschiebung von einer »begriffliche[n] « zu einer »visuellen Kultur«, bzw. vom lesbaren zum »sichtbaren Geist « diagnostizierte. ${ }^{3}$ In allen drei Fällen handelt es sich um die Erzählung eines Leitmedienwechsels, bei dem eine primär schriftlich-buchstabengestützte Kultur von einer primär technisch-bildlich symbolisierenden und kommunizierenden Kultur abgelöst wird. >Photographische Alphabetisierung` wäre demnach ein visual literacy-Programm, damit diejenigen, die bisher nur schriftlich geschult wurden, auch die Zeichen, oder vielmehr die phototechnischen Bilder, über die Kommunikation künftig stattfinden wird, zu ılesen` imstande sind.

1 Für Kritik danke ich Jan Kedves, Christina Striewski, Daniel Weidner und Maria Zinfert.

2 László Moholy-Nagy: „Diskussion über Ernst Kallai’s Artikel `Malerei und Fotografie«, in: i 106 (1927), S. 233 f., hier S. 233. Die meines Wissens erste Identifikation der Quelle leistet Krisztina Passuth: »Moholy-Nagy et Walter Benjamin. Une Rencontre«, in: Cahiers du Musée National d'Art Moderne 5 (1980), S. 398-409. Vgl. ebd. S. 403.

3 Marshall McLuhan: The Gutenberg Galaxy. The Making of Typographic Man, London (Routledge \& Kegan Paul) 1962. Béla Balázs: Der sichtbare Mensch oder die Kultur des Films (1924), Frankfurt a.M. (Suhrkamp) 2001, S. 16. Sowohl McLuhan als auch Balázs stellen diesen Wandel als Restitution dar, als Rückkehr zur Fülle des Bildlich-Visuellen bzw. des GestischExpressiven nach einer Epoche schriftlicher Dominanz. Moholys Historiographie ist dagegen nicht restitutiv, sondern einfach auf den kommenden Wechsel perspektiviert. 
Die (grund)schulhaft-erzieherische Konnotation des Begriffs der Alphabetisierung, gepaart mit dem Verweis auf das ABC und Einmaleins als Unterrichtsfächer, erinnern dabei auch an Moholys Tätigkeit als Pädagoge am Bauhaus, wo er die dortige Grundlehre unterrichtete, also einen Basiskurs, in dem die künftigen Modernisten gestalterisches Wissen über die Ableitung von Formen aus den sensorisch erfassbaren Eigenschaften von Materialien beigebracht bekamen. ${ }^{4}$ Diese Form-/Materiallehre gehört insofern in den Referenzbereich des Begriffs der photographischen Alphabetisierung, als sie auch den Bereich des Lichtes umfasst, dessen "lichtfaktur" vor allem im Photogramm als "schwarz-weiss-wirkung der fotografischen schicht« in Erscheinung tritt (Abb. 1). ${ }^{5}$

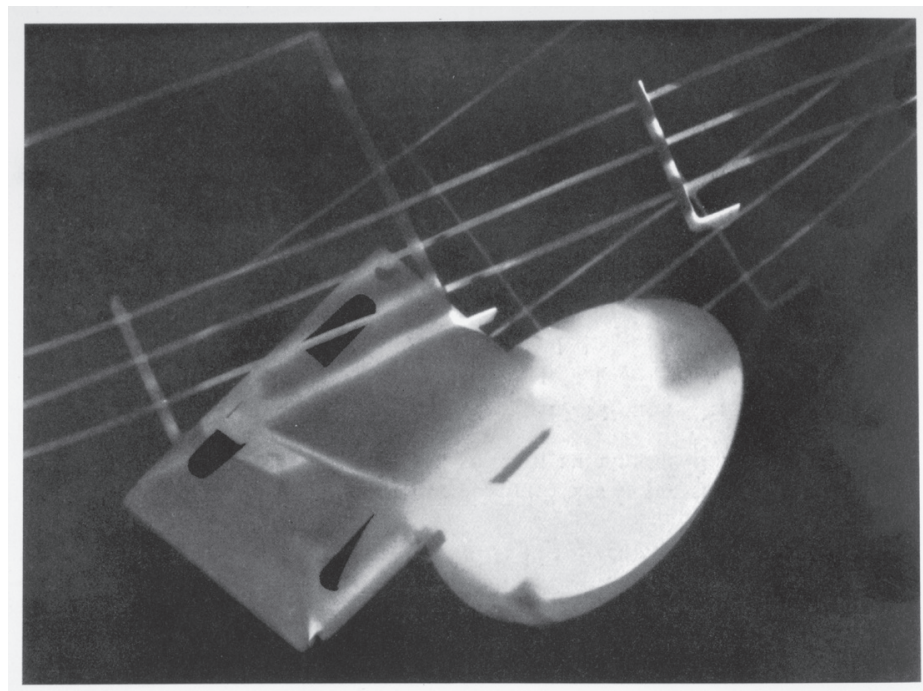

Abb. 1: Laszlo Moholy-Nagy: Fotogramm (1925). Illustration in: "Von Material zu Architektur» (1929) als Lichtfaktur-Beispiel.

Moholy zufolge wird hier künstlerischer Ausdruck »aus dem elementaren material der optischen gestaltung«, nämlich »aus dem direkten licht" realisiert, indem die photosensitiven Blätter "reflektorische [...] spiele [...] « aufzeichnen. ${ }^{6}$ Für ihn bildet dieser Typus der Formfindung die »brücke zu einer neuen optischen

4 László Moholy-Nagy: Von Material zu Architektur (1929), Mainz u.a. (Kupferberg) 1968. Zur Grundlehre vgl. S. 6 und den Begriff der vom Material ausgelösten »empfindungen« auf S. 21.

5 Moholy: "Diskussion« (Anm. 2), S. 234.

6 Die ersten beiden Zitate stammen aus: Moholy: Material (Anm. 4), S. 89, das letzte aus: ders. "Fotogramm und Grenzgebiet«, in: i 10 21/22(1928/1929), S. 190-192, hier S. 191. 
gestaltung", die sich von bestehenden künstlerischen Verfahren unterscheidet, weil sie im Gegensatz zu traditionellen künstlerischen Medien »das licht [...] in seiner direkten strahlung fluktuierend, oszillierend fast ohne umsetzung erfasst«, während ältere Techniken wie z. B. "pinsel« oder »farbstoff» Luminiszenz nur indirekt, in »sekundärste[r] materialisation « für künstlerische Zwecke verwenden können.?

Moholy dekliniert also drei Rückführungsbewegungen: die Rückführung von künstlerischen Formen auf sinnlich erfassbare Materialeigenschaften, die Rückführung der Lichtgestaltung in einen Zustand der beinahen Umsetzungslosigkeit, und schließlich die Rückführung visueller Gestaltung überhaupt auf das Element, das ihre Perzeption erst möglich macht, nämlich Licht. Diese Operationen verschaltet er in mustergültiger Avantgarderhetorik mit der Behauptung, dass die Kunst auf diesem Weg in einen neuen historischen Abschnitt eintritt, für den nun die Grundlagen zu schaffen seien. So tituliert er das kinetische Spiel luminoser Formen im abstrakten Animationsfilm Symphonie diagonale des Regisseurs Viking Eggeling als " $\mathrm{ABC}$ der Bewegungsfänomene in Hell-Dunkel« (Abb. 2-4). ${ }^{8}$

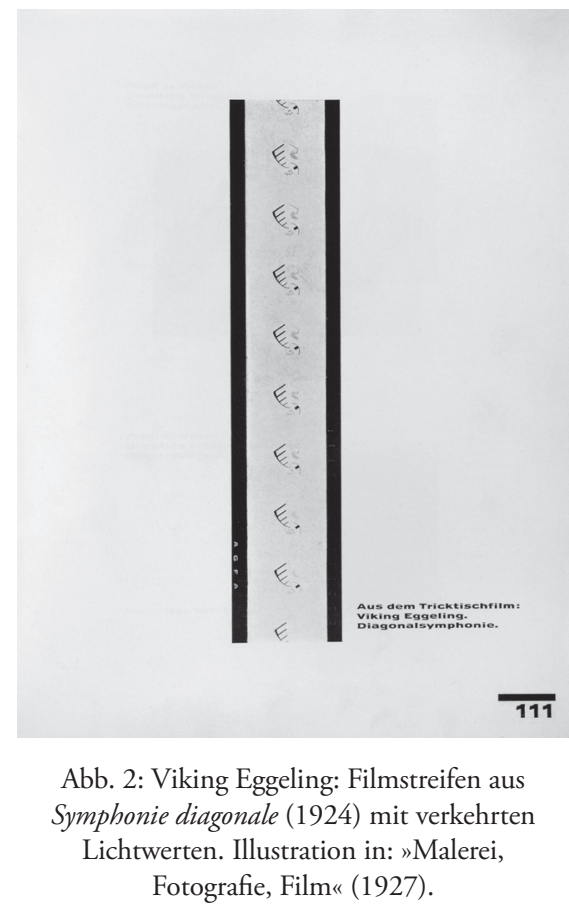

7 Ebd.

8 László Moholy-Nagy: Malerei, Fotografie, Film (1927), Reprint, Mainz u.a. (Kupferberg) 1967, S. $18 \mathrm{f}$. 


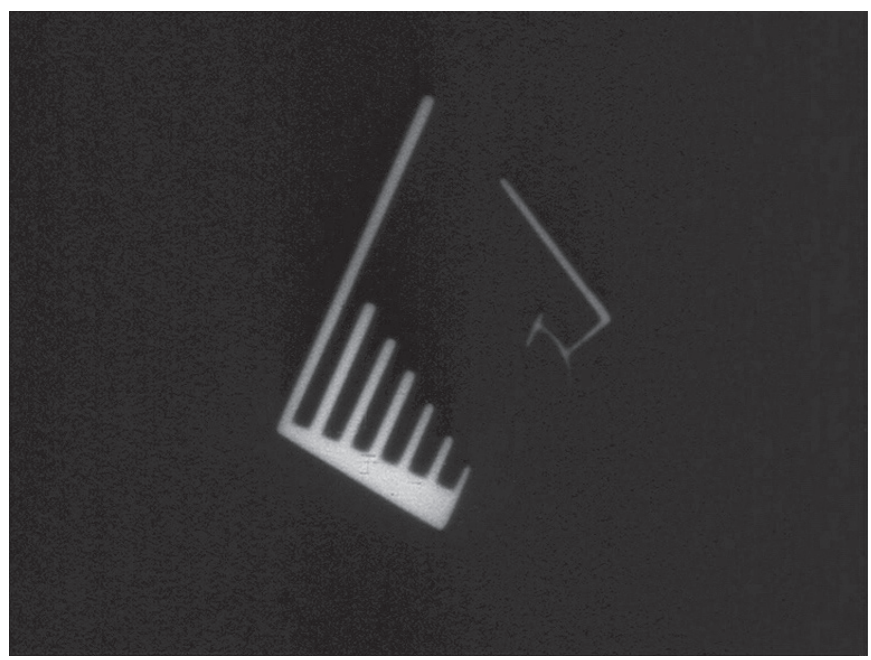

Abb. 3 (oben) und Abb. 4 (unten): Viking Eggeling: Standbildaufnahmen aus Symphonie diagonale (1924).

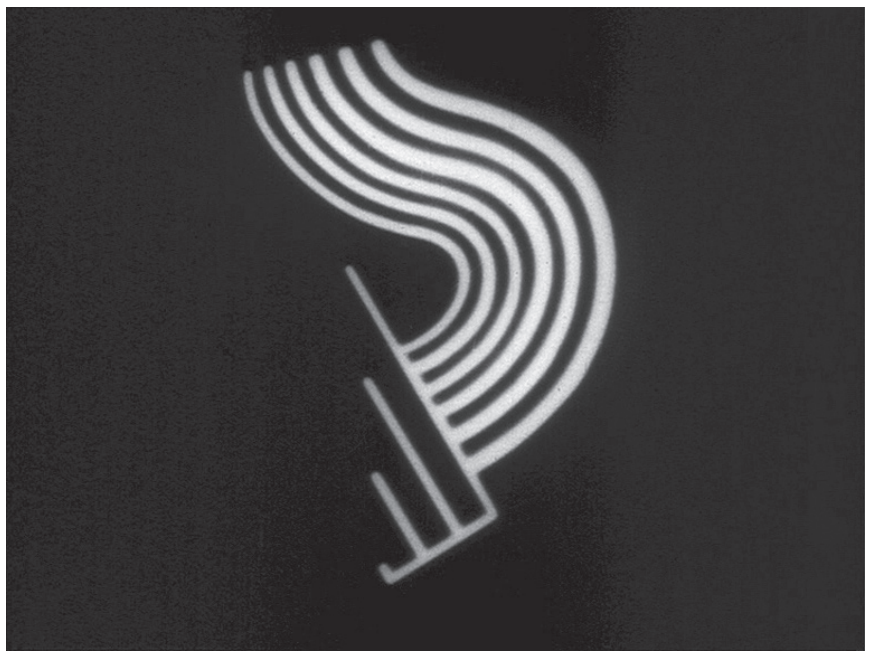

Eggelings auf dem schwarzen Feld der Leinwand zentrierte, einander überlagernde und ablösende Lichtzeichnungen, seine kammartig ausfächernden und in Kurven gebogenen Strichmuster antizipieren damit als abstrakte, fremdartige Zeichen das Vokabular einer kommenden Kunst, die aus verzeitlichten Figuren der Luminiszenz besteht. Wer wie Eggeling Licht im abstrakten Film zum Laufen bringt, oder wer im Bauhausgrundkurs das ABC photogrammatischer Formfindung aus den Bedingungen des Leuchtens erlernt, entwickelt nach 
Moholy gleichzeitig das Alphabet kommenden Gestaltens. Elementarer Kunsthochschulbesuch und Arbeit an den Elementen zukünftiger Kunst(geschichte) fallen damit in eins.

\section{Bestimmung der Rezeption}

Benjamins eingangs zitierter Bezug auf Moholys Diktum - "Nicht der Schrift-, sondern der Photographieunkundige wird, so hat man gesagt, der Analphabet der Zukunft sein" - fügt nun dessen Schlüsselbegriffen >Photographieunkundiger und `Analphabet der Zukunft mit dem Term des sSchriftunkundigen ein Element hinzu, das im Ausgangstext nicht enthalten ist. Der Kontrast, den diese interpolierende Geste zwischen Photographie und Schrift aufbaut, bereitet die Sätze vor, die sich in der Kleinen Geschichte dann unmittelbar anschließen: "Aber muß nicht weniger als ein Analphabet ein Photograph gelten, der seine eigenen Bilder nicht lesen kann? Wird die Beschriftung nicht zum wesentlichsten Bestandteil der Aufnahme werden?« (GS II, 385) Mit diesem Zusatz verschiebt Benjamin Moholys Modell in dreierlei Hinsicht. Erstens kappt er die Synonymie von sphotographischem Analphabetismus` und der Unfähigkeit, photographische Bilder zu lesen, indem er den Photographen, der seine Bilder nicht lesen kann, vom generell photographisch Illiteraten unterscheidet. Zweitens neutralisiert Benjamin die in Moholys Modell angelegte Chronologie einer substitutiven Mediensukzession, nach der eine photographische eine schriftliche Kultur beerbt. Entzifferungstätigkeit und Schriftbegriff behalten für Benjamin weiterhin ihre Gültigkeit und beziehen sich im starken Sinne jetzt auf photographische Bilder. Damit treten die Terme Lichtbild und Schrift in ein temporal beigeordnetes Verhältnis, nicht in eins der historischen Dominanzabfolge. Benjamin verabschiedet schließlich drittens Moholys Konzept der Photobildlichkeit, das sich aus der sensorischen Erfahrung der Lumineszenz herleitet, und ersetzt es durch das Paradigma der beschrifteten Aufnahme. Damit bezieht er sich zunächst auf die ganz konkrete zeitgenössische Praxis, die explodierende Zahl von Photographien, die in illustrierten Magazinen abgedruckt wurden, mit erläuternden Kommentaren und Bildunterschriften zu versehen. ${ }^{9}$

Im Gegensatz zu Moholy koppelt Benjamin die Terme Lichtbild und Schrift also nicht, um eine metaphorische Beziehung zwischen ihnen herzustellen, in der der Begriff des Alphabets auf den Bereich der Photographie übertragen wird, die somit zu einem allgemeinen Zeichensystem erklärt wird. Er kombiniert die Begriffe auch nicht im substitutiven Sinn, um die historisch-hegemoniale

9 Vgl. GS I, 485. 
Sukzession von Lichtbild auf Schrift auszudrücken. Stattdessen verwendet er ein Modell der räumlichen Kombination von Lichtbildern und sie begleitenden Buchstaben-Supplementen, die hier Lesbarkeit überhaupt erst herstellen. Aus dieser räumlichen Ko-Präsenz, die ganz konkret die Zusammenstellung von Schrift und Bild auf ein und derselben (gedruckten) Seite meint, erklärt sich auch der Begriff der Beschriftung, "ohne die alle photographische Konstruktion im Ungefähren stecken bleiben muß« (alle Zitate GS II, 385).

Indem Benjamin die Funktion der Bild-Beschriftung hier als Behelfsmittel gegen das ,Ungefähre konzipiert, fasst er gleichzeitig näher, was er unter der auf diese Weise herzustellenden Lesbarkeit photographischer Bilder versteht. Im Kunstwerkaufsatz beschreibt er die Wirkung der "Beschriftung« photographischer Aufnahmen in illustrierten Zeitungen - im Gegensatz zum Charakter des "Titel[s] eines Gemäldes" - als den eines »Wegweiser[s]«, der dem Betrachter "einen bestimmten Weg« zu den Bildern deutet (GS I, 485). Benjamin wählt das Adjektiv ১bestimmt hier nicht, um einen in seiner Partikularität zwar verbürgten, in seinem Wesen aber unbekannten Bildzugang zu bezeichnen, wie es die umgangssprachliche Verwendung des Wortes ‘bestimmt anzeigt, etwa wenn von `einer bestimmten Lösung`, einer bestimmten Person` o.ä. gesprochen wird. Die Bedeutung ergibt sich im Zusammenhang des Kunstwerkaufsatzes vielmehr daraus, dass Benjamin dort die "Rezeption in bestimmtem Sinne" der "freischwebende[n] Kontemplation" gegenüberstellt, die den Photographien nicht angemessen sei (ebd.). Damit ist erstens der Effekt der Beschriftung der Photographie als bestimmend definiert; zweitens wird die Rezeption, d.h. in diesem Fall die Betrachtung der Bilder als Wirkungsbereich dieser Bestimmung angegeben. Auch hierin unterscheidet sich Benjamins von Moholys Modell. In letzterem wird der historische Übergang der Rezeption von Buchstaben in diejenige von Photographien beschrieben. Moholy stellt also eine Sequenz der Gegenstände möglicher Rezeptionsakte dar, ohne dabei aber eine Aussage über die Qualität dieser Akte zu treffen. Nach dem Kunstwerkaufsatz hingegen situiert sich Bestimmung ausschließlich auf dieser qualitativen Ebene und ist auch nicht mit der Zuweisung einer bestimmten Bedeutung an die Lettern/ Lichtbilder-Kombinationen zu verwechseln. Benjamin bemerkt ausdrücklich, dass die Rezeptionswegweiser, die durch die Beschriftung der Photographie in den Illustrierten aufgestellt werden, zwar "obligat" geworden seien, dass es aber auch "gleichviel« sei, ob diese »[r]ichtige oder falsche» seien (ebd.). Bestimmung bezieht sich hier also tatsächlich nicht auf ein Objekt, das durch die Rezeption in irgendeiner Weise bestimmt würde, sondern allein auf den Akt der Rezeption selber. Oder, exakter: Die Formulierung, mit der im Kunstwerkaufsatz die »Rezeption in bestimmtem Sinne benannt wird, meint einen Rezeptionsmodus, der sich über einen Sinn - ein Fühlen, Sehen, Rezipieren, Aufnehmen etc. vollzieht, der als Sinn bestimmt ist. 
Benjamin beschreibt diese Rezeptions-Bestimmung zunächst als einen generellen Effekt der Beschriftung der Photographie, d. h. der Kombination von Buchstaben und Lichtbildern überhaupt. Ein in der Kleinen Geschichte beigefügter Zusatz, nach dem die Beschriftung die Photographie einer generellen »Literarisierung aller Lebensverhältnisse« (GS II, 385) zuschlägt, verweist allerdings auch auf eine spezifische künstlerische Praxis, auf die sich diese bestimmende Operation berufen kann. Den Begriff entlehnt Benjamin der Brecht'schen Bühnentheorie, die mit Literarisierung der Bühne ein Arrangement bezeichnet, in dem »Sprüche, Photographien und Sinnbilder" sich im Bühnenraum um und zwischen die "agierenden Personen " gruppieren. ${ }^{10}$ Literarisierung ist dabei zumindest in Teilen buchstäblich zu verstehen, denn eines ihrer zentralen Mittel, welches Brecht gemeinsam mit dem Bühnenbildner Caspar Neher entwickelte, bestand in betitelnden und erläuternden Schrift-Sätzen, die entweder auf Tafeln in den Bühnenraum hinabgelassen oder als Kurztexte von Scheinwerfern direkt in diesen hineinprojiziert wurden. ${ }^{11}$ Die Analogie, die Benjamin zwischen den beschrifteten Aufnahmen der illustrierten Magazine und der literarisierten Bühne des epischen Theaters herstellt, ist also insofern konkret, als in beiden Fällen ein bildliches Element, die Photographie bzw. die Szenerie des Bühnenraums, durch beigefügte Lettern modifiziert wird. Literarisierung bedeutet dabei nach Brecht »das Durchsetzen des `Gestalteten mit `Formuliertem « ${ }^{12}$ Die Lettern durchsetzen den visuellen Raum der Bühne, buchstäblich, sie durchmischen und durchdringen ihn. ${ }^{13}$

10 Bertolt Brecht: „Über die Literarisierung der Bühnen«, in: ders.: Schriften zum Theater, hg. v. Werner Hecht, Frankfurt a. M. (Suhrkamp) 1963-1964, Bd. 3, S. 253-255, hier S. 254.

11 Vgl. Vana Greisenegger-Georgila: "Caspar Nehers dialektische Bühne für Brecht«, in: Michael Schwaiger (Hg.): Bertolt Brecht und Erwin Piscator. Experimentelles Theater im Berlin der Zwanzigerjahre, Wien (Brandstätter) 2004, S. 73-95, hier S. 83, 85-87.

12 Bertolt Brecht: "Anmerkungen zur ,Dreigroschenoper«, in: ders.: Schriften zum Theater, hg. v. Werner Hecht, Frankfurt a. M. (Suhrkamp) 1963-1964, Bd. 2, S. 89-106, hier S. 90. Vgl. dort ebenfalls den ausdrücklichen Hinweis auf die Projektionstechnik. Benjamins Zitat dieser Formulierung steht in GS II, 524.

13 So weit abzusehen ist, lässt Benjamin im Unklaren, in welcher Beziehung die spezifische Praxis der Brecht/Neher'schen-Bühne zur diagnostizierten Literarisierung aller Lebensverhältnisse steht. Denn wenn ohnehin das Prinzip der beschriftenden Bestimmung auf breiter historischer Front greift, warum dann noch eine spezifische Theaterästhetik, die sich dessen durchsetzendem Effekt verschreibt? Vgl. auch Brigitte Werneburgs Hinweis, dass Benjamins Theorie der Beschriftung photographischer Bilder allgemein bleibt und damit zumindest hinsichtlich der zahlreichen partikularen Schrift/Bild-Montagepraktiken seiner Epoche hinreichend unbestimmt ist. Vgl. Brigitte Werneburg: „Ernst Jünger, Walter Benjamin und die Photographie. Zur Entwicklung einer Medienästhetik in der Weimarer Republik«, in: HansHarald Müller/Harro Segeberg (Hg.): Ernst Jünger im 20. Jahrhundert, München (Fink) 1995, S. 39-57, hier S. 48. 


\section{Exkurs: Bestimmte Zeit - das Lichtbild in der Geschichte}

In seinen letzten Schriften nimmt Benjamin das Konzept einer Entzifferung bzw. Lektüre der Bilder auf und spricht dort vom "gelesene[n] Bild« bzw. bekanntermaßen vom Bild als "Dialektik im Stillstand" (GS V, 577). Anders als im Fall der Photographie, deren Lesbarkeit vom heraufziehenden historischen Zustand einer Literarisierung sämtlicher Lebensverhältnisse, bzw. von der obligatorisch werdenden Beschriftung der Aufnahme abhängt, knüpft Benjamin das Lesbarwerden der Bilder in seinen spätesten Texten zunächst an eine innerhistorische Konjunktion zwischen einem gegenwärtigen und einem ihm diskontinuierlichen, vergangenen Zeitpunkt. Hiermit koppelt er den Begriff des Bildes an den des »Jetzt einer [...] Erkennbarkeit" (578). Mit diesem Wandel geht eine Verschiebung in der Korrelation der Terme Licht, Bild und Geschichte einher. Während die Kleine Geschichte der Photographie den Lichtbildern und ihrer Rezeption eine zeitliche Situierung gab, überträgt Benjamin im Passagenwerk eine Terminologie der Lichtphänomene in den Raum der Geschichte selbst, um die historisch-epistemologische Operation, die dialektisches Bild heißt, zu beschreiben: "Nicht so ist es, daß das Vergangene sein Licht auf das Gegenwärtige oder das Gegenwärtige sein Licht auf das Vergangene wirft, sondern Bild ist dasjenige, worin das Gewesene mit dem Jetzt blitzhaft zu einer Konstellation zusammentritt" (ebd.). Oder, in den Geschichtsthesen: "Nur als Bild, das [...] im Augenblick seiner Erkennbarkeit [...] aufblitzt, ist die Vergangenheit festzuhalten" (GS I, 695). Während die Kleine Geschichte also ein Narrativ über den historischen Einsatz der Lichtbilder präsentiert, wird im Passagenwerk und in den Geschichtsthesen das Lichtbild selber zu einer Instanz, die ein historisch-zeitliches Verhältnis artikuliert. Die Terme Konstellation und Aufblitzen stammen dabei ursprünglich aus der Beschreibung einer künstlerischen Lichtbildtechnik, nämlich einem Text Tristan Tzaras, der unter dem Titel Man Ray - La photographie à l'envers das Album Champs délicieux eingeleitet hatte, in welchem Man Ray eine erste Serie seiner Photogramme publizierte. Benjamin übersetzte dieses kurze Prosastück im Jahr 1924 für die von Mies van der Rohe, Ludwig Hilberseimer und anderen herausgegebene Zeitschrift $G$-Zeitschrift für elementare Gestaltung (Abb. 5 u. 6). 
Abb. 5: Tristan Tzara: Man Ray-Die Photographie von der Kehrseite. Übers. W. Benjamin, in: G - Zeitschrift für elementare Gestaltung 3 (1924).

Abb. 6: Als Illustration Man Ray: Rayographie (1922), in: ebd.
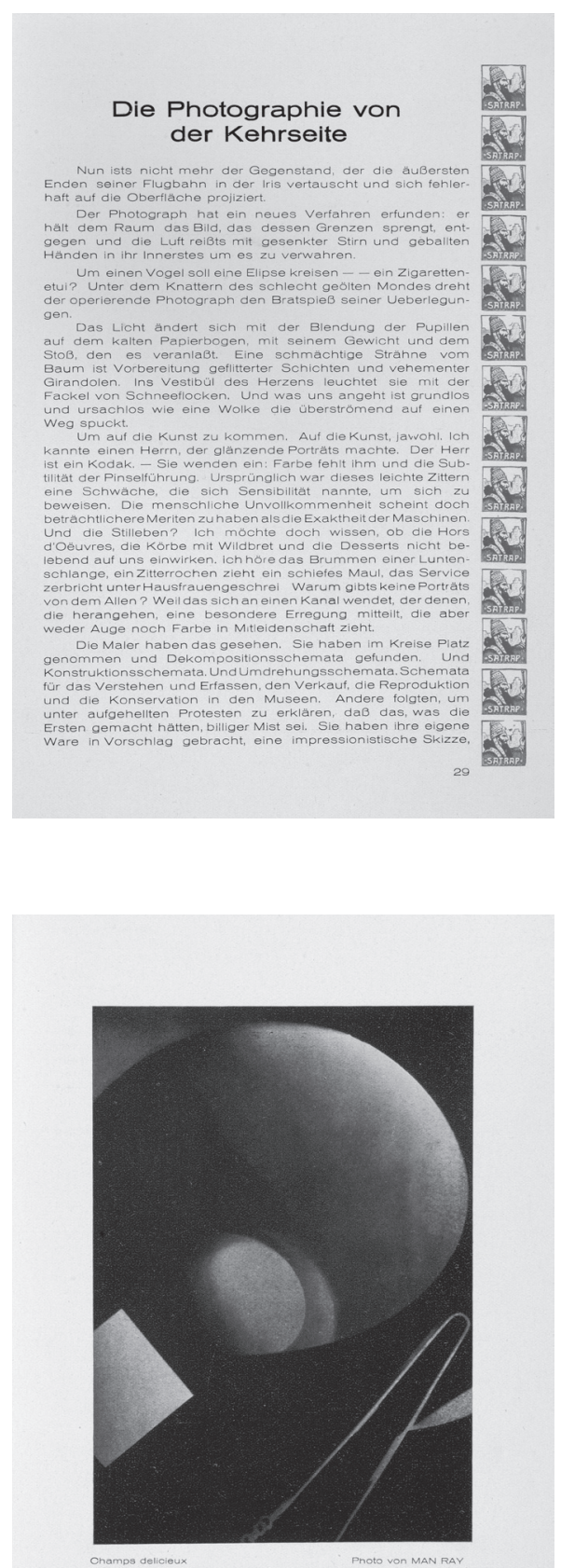
Im Original heißt es bei Tzara über Man Ray: »Il avait inventé la force d'un éclair tendre et frais, qui dépassait en importance toutes les constellations destinées à nos plaisirs visuels.« In Benjamins deutscher Fassung: „Er hatte die Tragweite eines zarten unberührten Aufblitzens entdeckt, die wichtiger war als alle Konstellationen, die uns zur Augenweide gestellt werden« (GS Suppl. I, 11 f.). Hier werden Aufblitzen und Konstellation also auf eine andere Weise kombiniert, als das in den späteren Schriften der Fall ist. 'Aufblitzen Emanation einer einzelnen Lichtquelle in der Dunkelkammer, die direkt vom photosensitiven Papier aufgenommen wird. Zu diesem Zeitpunkt steht diese aufscheinende Lichtfigur in Benjamins Denken auch noch allen Konstellationen, die uns zur Augenweide gestellt werden entgegen. In den PassagenwerksNotaten ist es dann die Konstellation selber, die aufblitzt und damit die Relation zweier diskontinuierlicher historischer Momente herstellt, die als verschiedene Lichtquellen figurieren, wie sie z. B. in einem Sternbild gegeben wären. In der Benjamin-Forschung ist wiederholt eine Ähnlichkeit zwischen der Beschreibung des dialektischen Bildes und den technischen Bedingungen photographischer Bildregistrierung konstatiert worden. Der Vergleich ruht erstens auf einer Verknüpfung zwischen dem Motiv des Lichtblitzes, wie es bei Benjamin vorkommt, und der Beleuchtung des filmischen Negativs. Seine zweite Säule bildet eine Parallelisierung zwischen dem arretierenden Charakter des Photos und der sistierenden Struktur des dialektischen Bildes, das ein jeweiliges historisches Jetzt der Erkennbarkeit festhält und damit aus dem Zeitablauf herausschneidet. Folgerichtig hat man auch in einer Variation des Titels Über den Begriff der Geschichte von Benjamins Theses on the Photography of History gesprochen. ${ }^{14}$ Die Analogie überzeugt bis zu einem gewissen Grad, auch wenn der Vergleich nicht die dualkonstellative Struktur mit einbezieht, die das dialektische Bild ausmacht. Zumindest im Stadium einer Notiz hat Benjamin allerdings einen Konnex zwischen Photographie und Geschichte hergestellt, der nicht auf eine strukturelle Parallele zwischen einem geschichtsphilosophischen Bildbegriff und einer visuellen Technologie hinausläuft. Stattdessen konstruiert Benjamin eine solche Verbindung auf der Ebene spezifischer künstlerischer und epistemologischer Praktiken, nämlich in der elliptischen Aufzeichnung "Das surrealistische Photo als photographierte Geschichte«, die sich unter seinen Nachlasspapieren findet. ${ }^{15}$ Ein erster Ansatz zur Entschlüsselung dieser Sentenz könnte von der Beobachtung ausgehen, dass Benjamin an surrealistischen Werken besonders interessierte, wie diese auf die geschmacklich und historisch diskreditierte Ding- und Bildwelt des

14 Vgl. Eduardo Cadava: Words of Light. Theses on the Photography of History, Princeton (Princeton University Press) 1997, S. XVIII, 3 f., 59-63. Vgl. dazu auch schon Norbert Bolz: „Der Fotoapparat der Erkenntnis", in: Fotogeschichte 9 (1989) 32.

15 WBA, Ms 1135. - Hier und im Folgenden zitiert nach den Siglen des Benjamin-Archivs in der Akademie der Künste, Berlin. 
19. Jahrhunderts zugriffen. Dem von anderen als "Haut-goût-Reiz« deklassierten appeal der Décors der untergegangen Epoche begegneten die Surrealisten mit ästhetischer Hingabe, die Benjamin zufolge die Qualität einer "Fixierung" (GS V, 572) erreicht. Als Beispiel könnte hier Max Ernsts Verwendung von Illustrationen und Drucken des 19. Jahrhunderts für seine Collagen-Romane wie Une semaine de bonté gelten oder, aus dem direkten Umkreis Benjamin'scher Interessen, Louis Aragons Begeisterung für die dem Verfall anheimgegebene Passage de l'Opéra. ${ }^{16}$ Durch solche künstlerischen Praktiken stellten die Surrealisten eine Verbindung zwischen ausgesuchten Bestandstücken der stilistischen Abraumhalde der Geschichte und ihrer eigenen Gegenwart her, die der historischen Konstellation, wie sie das dialektische Bild generiert, vergleichbar ist. Diese Art der Verbindung von Vergangenheit und Gegenwart liegt aber auf der Ebene künstlerischer Praktiken und positioniert sich zum historischen Wandel des Geschmacks und des jeweiligen affektiven Investments in ästhetische Phänomene; sie hat zunächst nichts mit der Struktur der Photographie als technisches Medium zu tun. ${ }^{17}$

Der Bezug zu einer zeitlich entfernten Epoche, wie ihn der surrealistische Geschmack zwischen den späten 1920er Jahren und dem 19. Jahrhundert stiftet, grundiert schließlich auch die Bedeutung des Begriffs der Bestimmung, wie ihn Benjamin in seiner Theorie des dialektischen Bildes gebraucht. Die getrennten historischen Momente verweisen dabei aufeinander. "[J]edes Jetzt", schreibt Benjamin, sei »das Jetzt einer bestimmten Erkennbarkeit» (578), und die Bestimmung der jeweiligen Erkennbarkeit ergibt sich daraus, dass jedes Bild »erst in einer bestimmten Zeit zur Lesbarkeit« (577) kommt. Diesen Zusammenhang nennt Benjamin den »historische[n] Index der Bilder«. Dieser zeigt nicht nur an, dass die Bilder »einer bestimmten Zeit angehören«, sondern auch dass diese bestimmte Zeit erst zu einem anderen bestimmten Zeitpunkt in einem konstellierenden Moment - einem Aufblitzen - lesbar wird (ebd.). ${ }^{18}$ Die Bilder der einen Epoche sind demnach nicht nur für eine andere Epoche bestimmt, sondern diese Zueignung definiert erst eine Gegenwart als solche: "Jede Gegenwart ist durch diejenigen Bilder bestimmt, die mit ihr synchronistisch

16 Ein genuin photographisches Beispiel wäre zu suchen. Der auf der Notiz in Klammern hinzugefügte Hinweis "Bifur" macht klar, dass Benjamin durchaus auch eine konkrete Bildquelle im Blick hatte, nämlich Photographien, die in der gleichnamigen Zeitschrift erschienen. Eine weitere in der Notiz erwähnte Quelle ist das Journal Variétés.

17 Vgl. allerdings Rosalind Krauss' Argument, dass zahlreiche künstlerische Verfahren des Surrealismus einer photographischen Logik folgen. Rosalind Krauss: »The Photographic Conditions of Surrealism", in: dies.: The Originality of the Avant-Garde and Other Modernist Myths, Cambridge, Mass. u. a. (The MIT Press) 1986, S. 87-118.

18 Zum Zusammenhang von Lektüre und Konstellation im Hinblick auf Benjamins Bildbegriff vgl. ausführlich Bettine Menke: Sprachfiguren. Name, Allegorie, Bild nach Benjamin, München (Fink) 1991, S. 288-294. Vgl. auch Anselm Haverkamp: Figura Cryptica. Theorie der literarischen Latenz, Frankfurt a. M. (Suhrkamp) 2002, S. 44 f., 50. 
sind " (578). So erst kann »eine bestimmte Epoche aus dem homogenen Verlauf der Geschichte« (GS I, 703) herausgesprengt und damit eben auch sbestimmt werden.

\section{Räumliche Bestimmung und reine Aufnahme - Zeichen und Mal}

Von der Kleinen Geschichte der Photographie über den Kunstwerkaufsatz bis zum Passagenwerk gehören Licht, Graphie und Bild damit in den späten Benjamin'schen Kanon. Allerdings hat Benjamin sehr viel eher begonnen, sich mit graphischen Elementen (Zeichnungen, Lettern, Diagrammen) und Lichterscheinungen auseinanderzusetzen, und zwar bereits im Rahmen seiner frühen Überlegungen zu Zeichen und Mal bzw. Zeichnung/Graphik und Malerei. Die Weise, in der dort beide Elemente aufeinander bezogen werden, bereitet die späteren Argumente vor; ihre Logik ist aber nicht deckungsgleich. Dort skizziert Benjamin auch Überlegungen zur Bestimmung im Feld visueller Phänomene, die von seinen späteren Texten abweichen. Tatsächlich lassen sich Bezüge auf dieses frühe Modell bis in seine späten Schriften hinein finden, das also weiterhin Gültigkeit hat.

Eine markante Kombination von Licht und Graphie erkennt Benjamin im Kontext seiner frühen Notizen in der Form des Menetekels, also der Feuerschrift, die auf einer der Mauern im Palast Belsazzars erscheint und mittels derer Gott - nach der biblischen Überlieferung - das nahende Ende von dessen Herrschaft ankündigt. ${ }^{19}$ Das "Ungeheure« dieser "Erscheinung", schreibt Benjamin, beruhe »zum großen Teil auf der nur Gott zuzuschreibenden Vereinigung" der ansonsten unvereinbaren "Gebilde« Zeichen und Mal (GS II, 605). Diese beiden Terme bilden dabei die Pole einer phänomenologischen Unterscheidung, die zwei Typen visueller Manifestation gegeneinander stellt. In seinen Notizen zur zweiten Fassung des Kunstwerkaufsatzes definiert Benjamin das »Phänomen [...], das man vielleicht als das `Mak im exaktesten Sinne [...] bezeichnen dürfte" als »eine farbige Konfiguration, die auf der Wand erscheint (aus ihr heraustritt oder auf sie geworfen wird)«(GS VII, 677). Diese ‘farbige Konfiguration` manifestiert sich - wie die Feuerschrift - auf dem Untergrund architektonischer Wandelemente und lässt sich als Erscheinung im eigentlichen Sinne beschreiben, als ein aufscheinendes Phänomen, dessen Sichtbarkeit sich

19 Vgl. im Alten Testament die Prophetenschrift Daniel, Kapitel 5. Der biblische Text selber scheint den Lettern an der Wand keine leuchtende Qualität zuzuschreiben, zumindest nicht ausdrücklich. Zu welchem Zeitpunkt dieser Aspekt als wesentlicher Zug des Menetekel-Topos in Erscheinung tritt, wäre zu ergänzen. 
in Abhängigkeit vom Zeitverlauf entwickelt. ${ }^{20}$ Außerdem ist sie an den Charakter der Farbigkeit gebunden. In allen drei Aspekten ist Benjamins Begriff des Mals kompatibel mit seiner Schilderung des Laterna-Magica-Lichtes im frühen Dialog Der Regenbogen (25). ${ }^{21}$ Auf Grundlage dieser phänomenologischen Basisbestimmung verteilen sich malartige Erscheinungen in Benjamins Schriften vom magisch-mystischen Bereich (z. B. das Menetekel) bis hin zu technischen Lichtprojektionsapparaten und der künstlerischen Technik der Malerei. Diese Skala fasst Benjamin in der Formel von der "Konfiguration, die magisch angesehen, transitorisch, profan angesehen eher transportabel zu nennen wäre« (677), die im Kontext seiner Überlegungen zur Malerei steht. Die profan-transportable Spielart des Mals bestünde demnach im zum Objekt gewordenen Tafelbild, das auf der vertikalen Struktur des architektonischen Trägers Wand auf- und wieder abgehängt werden kann, in dem das Aufscheinen und Vergehen des aufblitzenden Lichtes also zum Auftauchen und Verschwinden der auf den Rahmen gespannten Leinwand geworden ist. ${ }^{22}$ Als Beispiele für die Farbigkeit der Konfiguration nennt Benjamin neben der malerischen Leinwand auch die sich mischenden, vom ungrundierten Papier teilweise aufgesaugten Kolorierungen des "getuschte[n] Bild[es]" sowie die "feuchte Farbigkeit« der Verläufe des Laterna-Magica-Lichtes, die »möglichst

20 Vgl. auch die "Lichtreklame«, die Benjamin einmal einen neuen "Typus von Schrift" genannt hat. WBA, Ms 1109.

21 Vgl. auch Uwe Steiner: Die Geburt der Kritik aus dem Geiste der Kunst. Untersuchungen zum Begriff der Kunstkritik in den frühen Schriften Walter Benjamins, Würzburg (Königshausen \& Neumann) 1989, S. 48, 64. Steiner unterstreicht dort die Wichtigkeit von Baudelaires Schriften für die Farbtheorie des Regenbogen-Dialogs. Vgl. ebenfalls Heinz Brüggemann: Walter Benjamin über Spiel, Farbe und Phantasie, Würzburg (Königshausen \& Neumann) 2007, S. 170-174. Brüggemann schließt sich Steiner an, spezifiziert die Baudelaire-Referenz auf dessen Ausführungen zum Aquarell im Salon von 1846 und führt als weitere Quellen Jean Paul und Friedrich Theodor Vischer an. Vgl. schließlich Howard Caygill: Walter Benjamin. The Colour of Experience, London u. a. (Routledge) 1998, S. 82-89.

22 Benjamin erweitert diese Überlegungen mit Hinweis auf Ozenfant in Richtung der peinture murale, bei der das Tafelbild mit seinem architektonischen Träger - der Wand - verschmilzt. Vgl. GS VII, 672. Im dialektischen Gegenzug konstatiert Benjamin wiederum das historische Erodieren statischer Ausstellungsarchitekturen, die nun die Mobilität der Tafelbilder annehmen: „Die Ausstellungen nämlich, welche zum ersten Mal die Rezeption der Gemälde zur Sache der Massen machen, haben zum ersten Mal das Gemälde an Wänden ausgestellt, die von architektonischen Funktionen gänzlich entbunden waren« (675). Damit wäre das Mal in den architektonischen Träger eingegangen, der aber selber dessen transitorische Züge annimmt. Beide Tendenzen sind mit der Diagnose eines Architektonischwerdens der Künste in Zeiten historischer Übergänge zusammen zu denken, die der Kunstwerkaufsatz am Beispiel des Films entwickelt. Vgl. hierzu Philipp Ekardt: Splitting the Picture. Studies in Walter Benjamin's Image-Theory and in Alexander Kluge's Image-Practice, Yale University, PhD-Thesis 2009, S. 155-162. Zur Krisis der Malerei vgl. auch den Pariser Brief über Malerei und Photographie (GS III, 495-507). 
verschwommen, auflösend, ganz monoton nüanciert, ohne Licht- und Schattenübergänge« (25) erscheinen. Hier, so Benjamins Argument, finde durch die Abwesenheit von Chiaroscuro keine optische Modellierung von Volumina statt, und das Nichtvorkommen der graphischen Linie generiere auch nicht die für Räumlichkeit konstitutive Sukzession von Blatt als Untergrund und aufliegendem Strich. ${ }^{23}$ Dies sei hingegen beim Zeichen der Fall: „Die graphische Linie bezeichnet die Fläche und bestimmt damit diese indem sie sie sich selbst als ihrem Untergrund zuordnet" (GS II, 603), bzw.: "Die graphische Linie verleiht ihrem Untergrunde Identität« (604).

Benjamin entwirft damit das Mal im Gegensatz zum sich räumlich artikulierenden und Räumlichkeit auch erst herstellenden Zeichen als eine praktisch rein zeitliche Emanation von Farbe im Speziellen, Licht im Allgemeinen. ${ }^{24}$ An dieser Stelle ist auch Benjamins früher Begriff der (visuellen) Bestimmung verortet, der sich auf diesen spatialen Aspekt bezieht. Das Zeichen bestimmt etwas, weil es durch die Einschreibung der Linie auf dem liegenden Blatt ein Auf und Unter herstellt. Dies leisten die Erscheinungen des Mals in ihrer reinen luminosen Form offensichtlich nicht. ${ }^{25}$ Durch diese Kopplung des Begriffs der Bestimmtheit an die Kategorie der Räumlichkeit, die ihr Gegenstück in der nicht-bestimmenden Lichtmanifestation hat, wird die Verwandtschaft zwischen den Benjamin'schen Konzepten und jenen Termen deutlich, mit denen Heinrich Wölfflin zwei Typen der `Orientierung zu Sichtbarkeit` - nämlich lineare und malerische Modi - beschreibt, entlang derer er die Geschichte der westlichen Nachrenaissance-Kunst einteilt. Die Verbindung zu Wölfflin, dessen kunstwissenschaftliche Übung Benjamin während seiner Münchener Studiensemester besuchte, mag überraschen, denn es gilt als ausgemacht, dass Benjamin sich nach einer ersten jugendlichen Emphase praktisch durchgehend

23 Vgl. GS II, 606.

24 Vgl. aber die Variante: "Die Malerei proj[i]ziert den Raum in die vertikale Fläche" (GS VII, 675). Diese kurze Darstellung berücksichtigt nicht alle Aspekte der Benjamin'schen Theorie des Mals, die z.B. um die anthropologisch-leibliche Dimension einiger seiner Manifestationen, etwa im Erröten, zu ergänzen wäre. Vgl. dazu Sigrid Weigel: Walter Benjamin. Die Kreatur, das Heilige, die Bilder, Frankfurt a. M. (Fischer) 2008, S. $268 \mathrm{f}$.

25 Eggelings eingangs geschilderte Symphonie diagonale entkommt dieser Logik - ebenso wie der gesamte Bereich des Stummfilms, der leuchtende Titel und Dialogfragmente zwischen die Bildsegmente setzt; man fragt sich, ob die 'Neuheit des Schrifttypus der elektrischen Reklame für Benjamin ebenfalls in ihrer Kopplung von Licht und Lettern liegt. Vgl. Anm. 20. Was Benjamin anhand des Menetekels nur Gott zuzuschreiben bereit war, nämlich die Verbindung der Eigenschaften von Mal und Zeichen, scheint die Kino- und Werbetechnik spielerisch zu bewältigen. Zum Komplex der Film-Schrift in den frühen Jahren des Kinos vgl. das Kapitel "Hieroglyphics, Figurations of Writing", in: Miriam Hansen: Babel and Babylon. Spectatorship in American Silent Film, Cambridge/Mass. u. a. (Harvard University Press) 1991, S. 188-198. 
negativ zu dessen Arbeit geäußert hat. ${ }^{26}$ Tatsächlich besteht aber eine konzeptuelle Nähe zwischen Benjamins Begriffen Zeichen und Mal und Wölfflins Terminologie, weil der Kunstwissenschaftler - Benjamin nicht unähnlich - die Funktion des linearen, graphischen Modus als räumliche Bestimmung definiert: "Der lineare Stil ist ein Stil der plastisch empfundenen Bestimmtheit. Die gleichmäßig feste und klare Begrenzung der Körper gibt dem Beschauer eine Sicherheit, als ob er sie mit den Fingern abtasten könnte, und alle modellierenden Schatten schließen sich der Form so vollständig an, daß der Tastsinn geradezu herausgefordert wird. ${ }^{27}$ Gegen diese "Begreifung der Körper nach ihrem tastbaren Charakter - in Umriß und Fläche" setzt Wölfflin eine "Auffassung, die dem bloßen optischen Schein sich zu überlassen imstande ist und auf die sgreifbare Zeichnung verzichten kann «. ${ }^{28}$ Auf diese Weise permutiert Wölfflin die klassische Unterscheidung von disegno und colore bzw. pittura hin zur Unterscheidung von linearer bzw. zeichnerischer und malerischer Orientierung zur Sichtbarkeit. Das Benjamin'sche Begriffspaar Zeichen und Mal gehört insofern in diese Reihe, als es graphische und malerische, lineare und luminos-farbige Elemente, räumliche und nicht-räumliche, bestimmende und nicht bestimmende Modi miteinander kontrastiert. Diese werden bei Benjamin dann aber erstens von ihrer Beschränkung durch den engen stilistischen Kunstbezug befreit und auf das weite Spektrum möglichen visuellen Bedeutens - von der Magie bis zum Tafelbild - bezogen. Zweitens beschreiben sie, im Gegensatz zur Wölfflin'schen Kunstwissenschaft, keine Darstellungsstile, sondern eine sonderbare Kategorie visueller Signifizierungstechniken. Das Zeichen erzeugt räumliche Bestimmung nicht, weil es einen gezeichneten Gegenstand als räumlich fassbar darstellt, sondern weil die Interaktion von Linie und Untergrund ein räumliches Verhältnis und damit eine Bestimmung generiert, die im farbigen Licht des Mals nicht gegeben ist. Man wäre versucht, hier von einer Übertragung der Kategorie der Bestimmung auf die Ebene der Medien

26 Sigrid Weigel hat diese Ausschläge nachgezeichnet. Vgl. dies.: „Bildwissenschaft aus dem >Geiste der Philologie‘. Benjamins Wahlverwandtschaft mit der ıneuen Kunstwissenschaft und der Warburg-Schule«, in: Detlev Schöttker (Hg): Schrift Bilder Denken. Walter Benjamin und die Künste, Frankfurt a. M. u. a. (Suhrkamp, Haus am Waldsee) 2004, S. 112-127, hier S. 117. Die Benjamin-Forschung ist bis jetzt weitgehend der späteren Abqualifizierung des Wölfflin'schen Ansatzes durch Benjamin gefolgt, die sich z. B. aus der Rezension Strenge Kunstwissenschaft herauslesen lässt. Der Abwertung Wölfflins entsprach eine Aufwertung Riegls.

27 Heinrich Wölfflin: Kunstgeschichtliche Grundbegriffe. Das Problem der Stilentwicklung in der neueren Kunst, München (Bruckmann) ${ }^{7} 1929$, S. 23. Benjamin verfasste die Aufzeichnungen zu Zeichen und Mal entweder während oder kurz nach seinem Seminarbesuch bei Wölfflin. Zu Benjamin und Wölfflin vgl. ausführlicher Philipp Ekardt: „Benjamins Bekleidungsmodelle. Strumpf und Rüsche als Topologien der Bildtheorie«, in: Ingeborg Reichle/Steffen Siegel/ Achim Spelten (Hg.): Visuelle Modelle, München (Fink) 2008, S. 83-98, hier S. 88-92.

28 Wölfflin: Grundbegriffe (Anm. 27), S. 15. 
zu sprechen, mit denen in diesem Fall visuelle Medien (Graphik, Blatt, Licht, Projektionsapparat etc.) gemeint wären, wenn Benjamin nicht den Begriff des "Mediums« für die »Sphäre des Mals« (GS II, 605) reserviert hätte, die somit als jene Zone beschrieben ist, in der Bestimmung nicht stattfindet. ${ }^{29}$ Oder, wie Benjamin das rein farbige Medium im Regenbogen-Dialog beschreibt: Hier realisiert sich eine "raumlose Unendlichkeit der reinen Aufnahme" (GS VII, 25). ${ }^{30}$

Es werden also Kontinuitäten, aber auch wesentliche Verschiebungen von Benjamins früheren zu seiner späten Theorie deutlich. In beiden Fällen operiert Benjamin mit einem Konzept der nicht-objektbezogenen Bestimmung: Die Kombination von Photographie und Beschriftung, von der in den späteren Texten die Rede ist, erzeugt eine bestimmte Rezeption, nicht eine Bestimmung des rezipierten Gegenstandes; und der Räumlichkeit generierende Strich des Zeichens auf seinem Grund bestimmt nicht das Gezeichnete, sondern sorgt für eine Bestimmung des Zeichengrundes. In beiden Fällen sind es nicht die lichtbezogenen oder malerischen Modi, sondern die graphisch-linearen Elemente wie Zeichnung und Schriftzeichen, die diese Bestimmung hervorbringen. Und in beiden Fällen ist Bestimmung untrennbar mit Räumlichkeit verbunden. Sie entsteht im spatialisierenden Strich oder in der synchronen Zusammenstellung von Photo und schriftlichem Zusatz auf ein- und derselben Druckseite, die die zeitliche Sukzession von Photographie auf Schrift aushebelt, von der Moholy ausging.

Während die frühen Texte allerdings das räumlich-bestimmte Zeichen und das leuchtend-unbestimmte Mal als parallel existierende Kategorien beschreiben, entwickeln die späteren Schriften dann ein Modell, bei dem zumindest ein vermittelter Konnex zwischen Lichtphänomenen und Momenten der Bestimmung hergestellt wird. Die Scharnierfunktion erfüllt dort die Photographie, also wenn nicht projizierte, so doch lichtgenerierte Bilder, die die Rezeption bestimmen, indem sie mit Schriftelementen kombiniert werden. Wie der Begriff der Be-schriftung anzeigt, hat sich hier auch die bestimmende Funktion der Zeichen geändert, die nun nicht mehr auf ihren eigenen Untergrund bezogen sind, sondern auf ein ihnen außen Liegendes, nämlich die Lichtbild-Abdrucke in illustrierten Magazinen. Parallel zu dieser Umdeutung verschiebt sich bei Benjamin auch die Bedeutung des Begriffs der Aufnahme. In der Form der reinen Aufnahme, wie das pure Farbensehen im Regenbogendialog genannt wird,

29 Die "Sphäre des Zeichens«, schreibt Benjamin, sei hingegen "wahrscheinlich kein Medium» (GS II, 604).

30 Vgl. zum Begriff der reinen Aufnahme im Zusammenhang mit Benjamins Ästhetik der Entstaltung sowie der Praxis des Photogramms das Kapitel "A Dispersed, Spaceless Infinity of Pure Reception. Benjaminian 'Entstaltung , the Photogram, and the Appearance of the Medium«, in: Ekardt: Splitting (Anm. 22), S. 15-99. 
meint er den Zustand der aufnehmend-absorbierenden sensorischen Öffnung hin zu malartigen Erscheinungen. In der Kleinen Geschichte der Photographie geht aus dem Begriff einerseits das Konzept der beschrifteten Aufnahme hervor, bei dem sich die Bedeutung des Begriffs ,Aufnahmer fort von einer sensorischen Absorption durch das sehende Subjekt hin zum lichtempfindlichen Papier verschoben hat, das den Lichteindruck registriert. Der Aspekt der sensorischen Absorption, den der frühe Begriff der Aufnahme noch meinte, geht hier hingegen im Begriff der Rezeption auf, also der Prozessierung der Bilder, die, durch Beschriftung der Aufnahme - wie gezeigt - zur bestimmten Rezeption wird. 


\section{Abbildungsnachweise}

\section{Kathrin YaCaVone}

Die Photographien "zu ihrem Rechte kommen lassen«.

Zu Roland Barthes' Rezeption von Benjamins "Kleiner Geschichte der Photographie»

Abb. 1:

Le Nouvel Observateur: Spécial Photo, 2 (1977), S. 7. Bibliothèque nationale de France. Mit freundlicher Genehmigung von Robert Delpire für den Nouvel Observateur.

Abb. 2:

Die Literarische Welt, 38 (1931), S. 3. Deutsches Literaturarchiv Marbach.

(C) Hamburger Stiftung zur Förderung von Wissenschaft und Kultur/Suhrkamp Verlag.

Abb. 3:

Roland Barthes: La Chambre claire, S. 148 f. @ Éditions du Seuil, en coédition avec les Éditions de l'Étoile et Gallimard, 1980.

Abb. 4:

Le Nouvel Observateur: Spécial Photo, 2 (1977), S. 10 f. Bibliothèque nationale de France. Mit freundlicher Genehmigung von Robert Delpire für den Nouvel Observateur.

Abb. 5:

Roland Barthes: La Chambre claire, S. 74 f. @ Éditions du Seuil, en coédition avec les Éditions de l'Étoile et Gallimard, 1980.

Abb. 6:

Le Nouvel Observateur: Spécial Photo, 2 (1977), S. 18 f. Bibliothèque nationale de France. Mit freundlicher Genehmigung von Robert Delpire für den Nouvel Observateur.

\section{Philipp EKardT}

\section{Die Bestimmung der Aufnahme.}

\section{Licht und Graphie bei Walter Benjamin.}

Abb. 1

László Moholy-Nagy: Fotogramm (1925), verwendet als Illustration in: „Von Material zu Architektur« (1929), aus: ders.: Von Material zu Architektur, Mainz u. a. (Kupferberg) 1968, S. 89.

Abb. 2

Viking Eggeling: Filmstreifen aus Symphonie diagonale (1924) mit verkehrten Lichtwerten, verwendet als Illustration in: "Malerei, Fotografie, Film» 
(1927), aus: László Moholy-Nagy: Malerei, Fotografie, Film, Repr., Mainz u.a. (Kupferberg) 1967, S. 111.

Abb. 3 u. 4

Viking Eggeling: Standbildaufnahmen aus Symphonie diagonale (1924), 3 Min., $35 \mathrm{~mm}$.

Abb. 5 u. 6

Tristan Tzara: "Man Ray - Die Photographie von der Kehrseite«, übers. v. Walter Benjamin, in: G-Zeitschrift für elementare Gestaltung 3 (1924). Als Illustration Man Ray: Rayographie (1922), zuerst publiziert in: ders.: "Champs délicieux", in: G - Material zur elementaren Gestaltung 3 (1924), S. 29-31, Repr., München 1986.

\section{Arno Dusini}

\section{Das Buchstabieren Benjamins}

Abb. 1

Das große Nürnberg'sche ABC für Kinder in saubern Kupfern, sinnlich dargestellt; Nürnberg, in der Kaiserl Privilegierten Kunst und Buchhandlung bei A.G. Schneider u. Weigel [1803]. Aus der Sammlung Walter Benjamin. Faksimile-Druck, Frankfurt a. M. (Insel-Bücherei 945) 1970.

Abb. 2

"Danziger Lesekasten« aus dem Ausstellungskatalog Max Ernst in Köln. Die rheinische Kunstszene bis 1922, hg. v. Wulf Herzogenrath, Köln 1980, S. 225.

Abb. 3

Ausschnitt aus dem Brief Benjamins vom 12. Oktober 1939 an Gretel Adorno. Abdruck mit freundlicher Erlaubnis des Walter-Benjamin-Archivs der Akademie der Künste, Berlin (WB-GA_0049_12.10.39), S. 2.

Abb. 4

"Walter Benjamin an Gretel Adorno, 12. Oktober 1939«, in: Walter Benjamin, Gesammelte Briefe, Bd. 6: 1938-1941, hg. v. Christoph Gödde/Henri Lonitz, S. 341-344. (C) Suhrkamp Verlag, Frankfurt a. M. 1999.

\section{Malte Kleinwort}

\section{Zur Desorientierung im Manuskript der Vorrede zu Benjamins Trauerspielbuch}

Abb. 1

Ursprung des deutschen Trauerspiels (1924), Walter Benjamin Archiv der Jewish National \& University Library in Jerusalem; abgebildet: S. 2 (Ausschnitt). Vgl. GS I, 924-950 [Signatur: Arc.4* 1598/109]. Maßstab: 1:1,4. 